\title{
Long-term intraocular pressure changes after vitrectomy for epiretinal membrane and macular hole
}

\author{
Masato Fujikawa • Osamu Sawada • Masashi Kakinoki • \\ Tomoko Sawada $\cdot$ Hajime Kawamura $\cdot$ Masahito Ohji
}

Received: 8 May 2013 /Revised: 7 August 2013 / Accepted: 23 September 2013 /Published online: 8 October 2013

(C) The Author(s) 2013. This article is published with open access at Springerlink.com

\begin{abstract}
Background To investigate long-term intraocular pressure (IOP) changes after vitrectomy for epiretinal membrane (ERM) or macular hole (MH).

Methods We retrospectively reviewed the medical records of 57 eyes with ERM and 61 eyes with $\mathrm{MH}$ that underwent vitrectomy. IOP levels and changes at 1, 3, 6, 12 months, and the final visit from baseline were evaluated in vitrectomized eyes and non-vitrectomized fellow eyes.

Results In the ERM group, the mean follow-up period was 29.3 months; the mean preoperative IOP in the operated eyes was $12.9 \pm 2.5 \mathrm{mmHg}$ and the final IOP was $13.2 \pm 2.9 \mathrm{mmHg}$. In the $\mathrm{MH}$ group, the mean follow-up period was 25.6 months; the mean preoperative IOP in the operated eyes was $13.3 \pm$ $2.5 \mathrm{mmHg}$ and the final IOP was $14.0 \pm 3.2 \mathrm{mmHg}$. The mean final IOP of the fellow unoperated eyes was $13.0 \pm 2.5 \mathrm{mmHg}$ in the ERM group and it was $12.9 \pm 3.2 \mathrm{mmHg}$ in the $\mathrm{MH}$ group. A significant difference was found between the operated eyes and fellow eyes at the final visit in the MH group $(P<0.01)$ but not in the ERM group $(P=0.40)$. MH group was significantly at high risk of IOP increase after vitrectomy $(P<0.01)$.

Conclusions IOP increase after vitrectomy was found in some eyes with MH during long-term follow-up but it was unlikely in eyes with ERM.
\end{abstract}

Keywords Intraocular pressure $\cdot$ Open-angle glaucoma . Vitrectomy $\cdot$ Epiretinal membrane $\cdot$ Macular hole

The authors have no proprietary interest in any aspect of this study. Supported in part by a grant from the Ministry of Education, Culture, Sports, Science and Technology of Japan (\#21592255) and a grant from the Ministry of Health, Labour and Welfare.

M. Fujikawa $(\bowtie) \cdot$ O. Sawada $\cdot$ M. Kakinoki $\cdot$ T. Sawada $\cdot$

H. Kawamura $\cdot$ M. Ohji

Department of Ophthalmology, Shiga University of Medical Science,

Seta Tsukinowacho, Otsu, Shiga 520-2192, Japan

e-mail: fujikawa@belle.shiga-med.ac.jp

\section{Introduction}

Transient increases in intraocular pressure (IOP) after pars plana vitrectomy due to inflammation, silicone oil tamponade, residual viscoelastic substance, and expansile gas filling are well-known early postoperative complications [1-6]. However, a few previous studies have reported late development of open-angle glaucoma (OAG) after vitrectomy [7-10].

Chang firstly suggested the development and worsening of OAG in patients who underwent vitrectomy in 2006 [7]. Luk et al. also reported the risk of OAG after vitrectomy in patients with epiretinal membrane (ERM) and idiopathic macular hole (MH) [8]. In contrast, Yu et al. reported that OAG does not develop after vitrectomy in patients with ERM, MH, and retinal detachment [9]. Lalezary et al. also reported that OAG does not develop after vitrectomy in patients with diabetes and vitreous hemorrhage, ERM, or MH [10].

These reports included various diseases, IOP measurement methods, and diagnostic criteria for OAG. In addition, the presence of diabetes might complicate this issue because of the potential for a decreased risk of OAG modified by retinal hypoxia [11, 12]. Vitrectomy for ERM is considered to be the least clinically invasive among the various vitrectomy procedures; therefore, a simple comparison between ERM and other vitreoretinal diseases for vitrectomy might reveal further mechanism of late-onset OAG. We performed a retrospective study to evaluate IOP changes after vitrectomy in eyes with ERM and MH including naive fellow eyes to evaluate the effect of the surgery on IOP changes.

\section{Patients and methods}

We conducted a retrospective review of the medical records of 190 patients with ERM and 157 patients with MH who underwent surgery performed by two surgeons (MO, HK) 
from May 2005 through November 2009. A three-port pars plana vitrectomy using a 20,23 , or 25 -gauge system under retrobulbar anesthesia was performed using the ACCURUS vitrectomy system (Alcon, Ft. Worth, TX). Simultaneous or two-stage cataract surgery (phacoemulsification and aspiration) was performed if needed. Postoperatively, steroidal eye drops (betamethasone $0.1 \%$ eye drops) were instilled for $1-$ 2 weeks followed by fluorometholone $0.1 \%$ for several weeks.

The inclusion criteria were a treatment-naive ERM or MH and at least a 12-month follow-up period including examinations at 1, 3, 6, and 12 months after vitrectomy. The exclusion criteria were a preoperative diagnosis of glaucoma or ocular hypertension (OHT); diabetes with or without diabetic retinopathy; and a history of vitrectomy, penetrating ocular trauma, ocular inflammation, ocular ischemia, or therapy with a long-term topical steroid medication. These criteria also applied to the fellow eyes. We also evaluated the preoperative lens status (phakia, pseudophakia, or aphakia) and the use of intraoperative adjuvant drugs such as triamcinolone acetonide to visualize the vitreous body or for membrane peeling and indocyanine green (ICG) stain to visualize the internal limiting membrane (ILM). After a fluid-gas exchange using $20 \%$ sulfur hexafluoride expansile gas, patients who underwent surgery for an $\mathrm{MH}$ were instructed to maintain a face-down position for 1 week postoperatively.

The IOP was measured three times at preoperative examination and follow-up visits after vitrectomy using a noncontact tonometer in the operated eye and fellow eye of each patient. Three IOP values were averaged and analyzed between baseline and each postoperative visit at 1, 3, 6, 12 months and the final visit. We defined IOP increase of $4 \mathrm{mmHg}$ as a cutoff point because the mean diurnal fluctuation is $3.7 \mathrm{mmHg}$ in normal eyes without glaucoma [13]. The best-corrected visual acuity (VA) was measured using a Landolt $\mathrm{C}$ chart test, and the value was converted to the logarithm of the minimum angle of resolution (logMAR). Statistical analyses were performed using GraphPad Prism 6 software (GraphPad Software Inc., La Jolla, CA) and JMP 10 software (SAS Institute Inc., Cary, NC). Student's unpaired and paired t-tests were used to compare two groups of normally distributed independent and matched groups, respectively. Mann-Whitney's U and Wilcoxon signed-rank tests were used to compare two groups of independent and matched groups without normal distribution, respectively. Multivariate analysis and Kaplan-Meier survival analysis with log-rank test were also performed. Data are expressed as the mean \pm standard deviation; $P$ values less than 0.05 were considered significant.

\section{Results}

A total of 57 eyes with ERM and 61 eyes with MH met the inclusion criteria. The mean follow-up period was $29.3 \pm$ 15.7 months (range, 12-61.3) in the ERM group and 25.6 14.1 months (12-57.7) in the MH group.

Table 1 shows the baseline demographics of the current study. There was no difference in the follow-up periods, gender distribution, or patient ages between the two groups. There was a significant difference in the preoperative logMAR VA $(P<0.0001)$.

Table 2 shows the surgical details. There was no difference between the two groups in the preoperative and postoperative lens status regarding the presence of the crystalline lens. The rates of intraoperative adjunctive procedures including use of triamcinolone acetonide, ICG, and ILM peeling were significantly higher in the MH group than in the ERM group $(P<$ 0.0001 , respectively).

In the ERM group, the mean preoperative IOP of the operated eyes was $12.9 \pm 2.5 \mathrm{mmHg}$ (range, 8.3-18.5) and the mean IOP at the final visit was $13.2 \pm 2.9 \mathrm{mmHg}$ (range, $8.0-19.0)$. Those in the fellow eyes were $13.1 \pm 2.5 \mathrm{mmHg}$ (range, 8.0-18.3), and $13.0 \pm 2.5 \mathrm{mmHg}$ (range, 8.5-19.3), respectively. No difference was found between the operated eyes and fellow eyes at baseline (paired $t$-test, $P=0.64$ ) and it remained insignificant to the final visit (paired $t$-test, $P=0.40$ ) (Fig. 1).

In the MH group, the mean preoperative IOP in the operated eyes was $13.3 \pm 2.4 \mathrm{mmHg}(8.0-18.7)$ and the mean IOP at the final visit was $14.0 \pm 3.2 \mathrm{mmHg}(6.3-21.7)$. Those in the fellow eyes were $13.3 \pm 2.5 \mathrm{mmHg}(8.3-19.0)$, and $12.9 \pm$ $3.2 \mathrm{mmHg}(6.7-24.0)$, respectively. No difference was found between the operated eyes and fellow eyes at baseline (paired $t$-test, $P=0.89$ ), however, the IOP only at the final visit in the operated eyes was significantly higher than fellow eyes in the MH group (paired $t$-test, $P<0.01$ ) (Fig. 2).
Table 1 The baseline demographics of epiretinal membrane (ERM) group and macular hole (MH) group

\begin{tabular}{llll}
\hline & $\begin{array}{l}\text { ERM } \\
n=57\end{array}$ & $\begin{array}{l}\text { MH } \\
n=61\end{array}$ & $P$ \\
\hline Operation date & $2005.5-2009.11$ & $2004.12-2009.11$ & - \\
Follow up period, months (range) & $29.3 \pm 15.7(12-61.3)$ & $25.6 \pm 14.1(12-57.7)$ & 0.19 \\
Male/female & $15(26 \%) / 42(74 \%)$ & $20(33 \%) / 41(67 \%)$ & 0.55 \\
Age, years & $66.0 \pm 9.4(32-84)$ & $65.1 \pm 7.3(44-82)$ & 0.36 \\
Best corrected visual acuity (logMAR) & $0.294 \pm 0.171$ & $0.708 \pm 0.311$ & $<0.0001$ \\
\hline
\end{tabular}


Table 2 Lens status and surgical procedures

${ }^{\text {a }}$ Preoperative existence of crystalline lens

${ }^{\mathrm{b}}$ Postoperative existence of crystalline lens

ERM epiretinal membrane; $M H$ macular hole

$T A$ triamcinolone acetonide; $I C G$ indocyanine green; ILM internal limiting membrane

\begin{tabular}{|c|c|c|c|c|}
\hline & & $\begin{array}{l}\text { ERM } \\
\mathrm{n}=57(48.3 \%)\end{array}$ & $\begin{array}{l}\mathrm{MH} \\
\mathrm{n}=61(51.7 \%)\end{array}$ & $P$ \\
\hline Preoperative lens status & $\begin{array}{l}\text { Pseudophakia } \\
\text { Aphakia }\end{array}$ & $\begin{array}{l}7(12.3 \%) \\
0(0.0 \%)\end{array}$ & $\begin{array}{l}2(3.2 \%) \\
1(1.6 \%)\end{array}$ & $0.19^{\mathrm{a}}$ \\
\hline Cataract surgery & $\begin{array}{l}\text { Combined PEA+IOL } \\
\text { Two-staged PEA+IOL } \\
\text { Lens spared }\end{array}$ & $\begin{array}{l}46(80.7 \%) \\
2(3.5 \%) \\
2(3.5 \%)\end{array}$ & $\begin{array}{l}57(93.4 \%) \\
0(0.0 \%) \\
1(1.6 \%)\end{array}$ & $0.61^{\mathrm{b}}$ \\
\hline Adjunctive procedure & $\begin{array}{l}\text { TA visualization } \\
\text { ICG staining } \\
\text { ILM peeling }\end{array}$ & $\begin{array}{l}21(36.8 \%) \\
3(5.3 \%) \\
9(15.8 \%)\end{array}$ & $\begin{array}{l}50(82.0 \%) \\
51(83.6 \%) \\
61(100.0 \%)\end{array}$ & $\begin{array}{l}<0.0001 \\
<0.0001 \\
<0.0001\end{array}$ \\
\hline
\end{tabular}

Table 3 shows the proportions of IOP increases by $4 \mathrm{mmHg}$ or greater compared with baseline. At the final visit, the IOP increased and sustained by $4 \mathrm{mmHg}$ or more from baseline in $4(7.0 \%)$ operated eyes and $4(7.0 \%)$ fellow eyes in the ERM group $(P=1.00)$ and $10(8.2 \%)$ operated eyes and $7(4.9 \%)$ fellow eyes in the MH group $(P=0.60)$. No significant differences were found between the two groups in the prevalence of IOP increases in the operated eyes at final visit $(P=0.16)$. IOP changes were statistically insignificant at any follow up period between eyes operated with and without TA.

We defined as the death event when the IOP increased by $4 \mathrm{mmHg}$ or greater than baseline in operated eyes and performed Kaplan-Meier survival analysis. The operated eyes in $\mathrm{MH}$ group showed statistically significantly higher risk of IOP increase than those in ERM group by log-rank test $(P<$ 0.01) (Fig. 3). Multivariate analysis revealed that age and baseline IOP alone significantly affected for the IOP value of operated eyes at final visit but not in disease. (Table 4)

During the follow-up period, no eye was diagnosed with glaucoma or treated with antiglaucoma drugs.

\section{Discussion}

The first report about late-onset OAG was by Chang in 2006, in which he suggested the development and worsening of

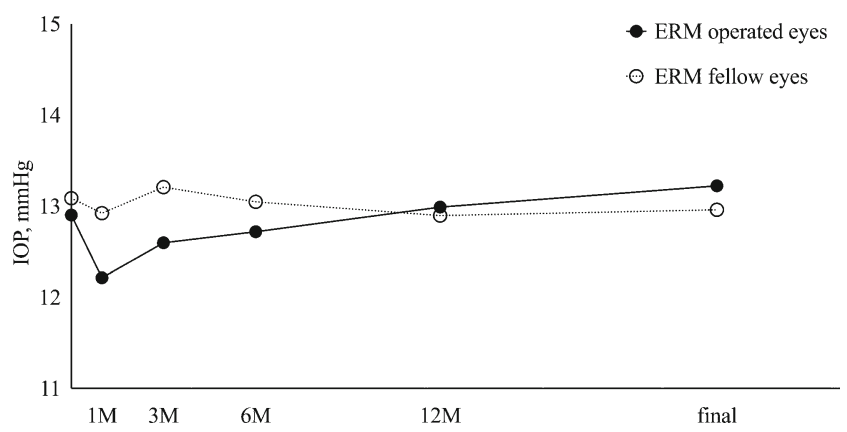

Fig. 1 Intraocular pressures (IOP) of operated eyes for epiretinal membrane $(E R M)$ and fellow eyes. No difference was found between the operated eyes and fellow eyes at the final visit $(P=0.40)$
OAG in patients who underwent vitrectomy and found that the mean IOP increased significantly in operated eyes compared with fellow eyes, especially in pseudophakic and aphakic eyes [7]. He suggested that the incidence of OAG after vitrectomy might increase to $15-20 \%$ and that the pathogenesis was assumed to be increased partial pressure of oxygen in the vitreous cavity after vitrectomy that might cause oxidative stress on the trabecular meshwork in the anterior chamber, particularly in the absence of a crystalline lens. Luk et al. also reported the risk of OAG after vitrectomy in patients with an epiretinal membrane (ERM) and idiopathic macular hole $(\mathrm{MH})$ [8]. Those investigators reported that the incidence of late-onset OAG was $7.9 \%$ in vitrectomized eyes and that $13 \%$ of pseudophakic eyes developed OAG; the incidence in phakic eyes was $2 \%$. The authors concluded that the crystalline lens might protect against late development of OAG after vitrectomy.

In contrast, Yu et al. and Lalezary et al. reported that OAG does not develop after vitrectomy. Yu et al. mainly analyzed patients with ERM, MH, and retinal detachment. They reported the prevalence rates of $\mathrm{OAG}$ and ocular hypertension were $4.31 \%$ and $4.31 \%$ in vitrectomized eyes and $2.49 \%$ and $2.95 \%$ in fellow eyes. The study concluded that vitrectomy was safe and did not increase the risk of OAG [9]. More recently, Lalezary et al. evaluated IOP after vitrectomy in patients with diabetes and vitreous hemorrhage, ERM, or

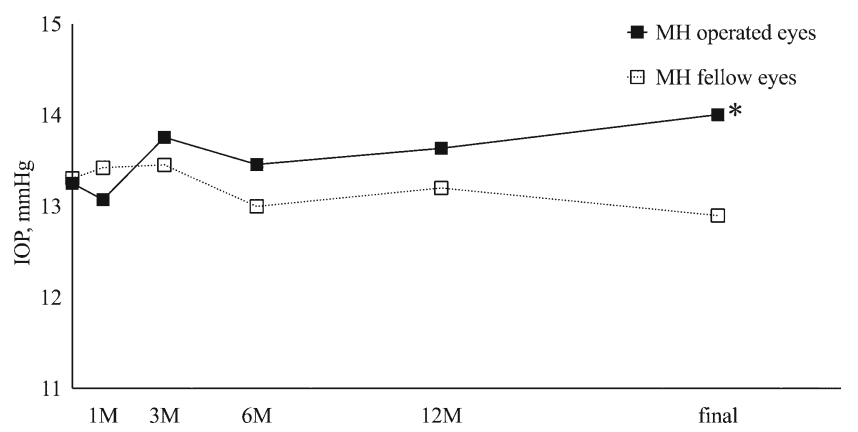

Fig. 2 Intraocular pressures (IOP) of operated eyes for macular hole $(\mathrm{MH})$ and fellow eyes. The IOP at the final visit in the operated eyes (asterisk) was significantly higher than fellow eyes $(P<0.01)$ 


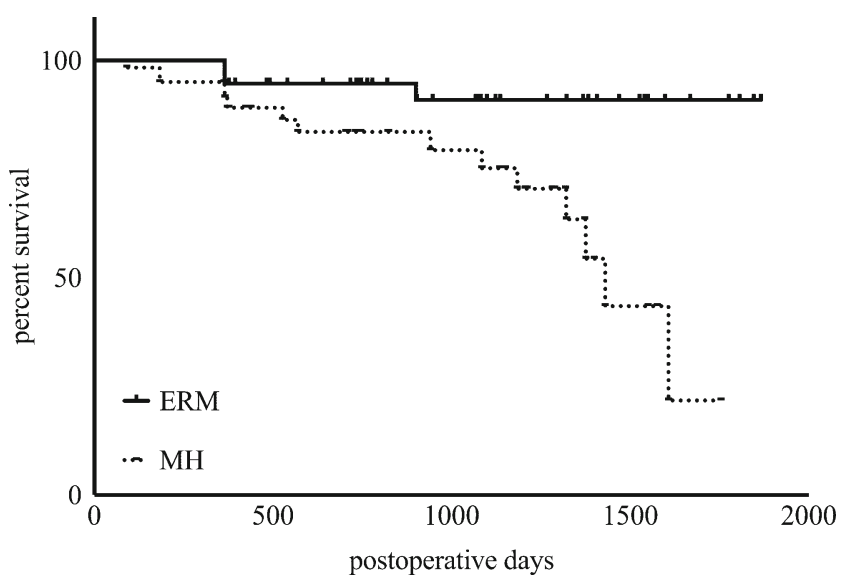

Fig. 3 The Kaplan-Meier survival curves in operated eyes for epiretinal membrane (ERM) (solid line) and macular hole $(\mathrm{MH})$ (dashed line) with postoperative intraocular pressure by $4 \mathrm{mmHg}$ or greater from baseline. MH had significantly at high risk of IOP increase after vitrectomy $(P<$ 0.01 by log-rank test)

$\mathrm{MH}$ and reported that the incidence of increased IOP levels exceeding $4 \mathrm{mmHg}$ was not significant between operated eyes $(15 \%)$ and fellow eyes $(14 \%)$. They concluded that the IOP does not increase after vitrectomy even after removal of the crystalline lens [10].

The four studies mentioned previously included various diseases, IOP measurement methods, and diagnostic criteria for OAG. In addition, the presence of diabetes might complicate this issue because of the potential for a decreased risk of OAG modified by retinal hypoxia [11, 12]. Vitrectomy for ERM is considered to be the least clinically invasive among the various vitrectomy procedures; therefore, a simple comparison between ERM and other vitreoretinal diseases for vitrectomy might shed light on the further mechanism of late-onset OAG.

When we compared the IOP values between the operated eyes and fellow eyes in the current study, we found no significant difference in the ERM group throughout the study period; however, we found a significant difference at the final visit in the MH group. Furthermore, the mean IOP increase in the operated eyes in the $\mathrm{MH}$ group was $0.7 \mathrm{mmHg}$, which was markedly higher than that of $0.3 \mathrm{mmHg}$ in ERM group. This difference suggests the potential risk of IOP increases and developing late-onset $\mathrm{OAG}$ in the MH group. It is difficult to define a clear criterion for diagnosis of late-onset OAG.

Table 3 The proportions of the intraocular pressure (IOP) increases $4 \mathrm{mmHg}$ or greater compared with baseline

\begin{tabular}{llr}
\hline & $12 \mathrm{M}$ & \multicolumn{1}{c}{ Final } \\
\hline ERM operated $\mathrm{n}=57$ & $3(5.3 \%)$ & $4(7.0 \%)$ \\
ERM fellow $\mathrm{n}=57$ & $0(0.0 \%)$ & $4(7.0 \%)$ \\
MH operated $\mathrm{n}=61$ & $5(3.3 \%)$ & $10(8.2 \%)$ \\
MH fellow $\mathrm{n}=61$ & $1(3.3 \%)$ & $7(4.9 \%)$ \\
\hline
\end{tabular}

Table 4 Multiple linear regression analysis to investigate the risk factors affecting postoperative IOP changes

\begin{tabular}{lcc}
\hline Variable & $\beta^{*}$ & $P$ \\
\hline Disease (ERM) & -0.16 & 0.4 \\
Gender (female) & -0.04 & 0.67 \\
Age & -0.36 & $<0.001$ \\
Lens status (aphakia) & -0.2 & 0.34 \\
Lens status (combined) & 0.09 & 0.58 \\
Lens status (lens spared) & -0.12 & 0.43 \\
System (20 gauge) & 0.02 & 0.89 \\
System (23 gauge) & -0.16 & 0.15 \\
TA (-) & -0.06 & 0.57 \\
ICG (-) & -0.11 & 0.43 \\
ILM peeling (-) & 0.21 & 0.24 \\
Baseline IOP & -0.29 & $<0.01$ \\
\hline
\end{tabular}

${ }^{*}$ Standardized coefficient; ERM epiretinal membrane; TA triamcinolone acetonide

$I C G$ indocyanine green; ILM internal limiting membrane; $I O P$ intraocular pressure

Hence, we excluded patients with diabetes who might be affected by relative ocular hypoxia and obtained the time series graph of the IOP levels from preoperatively to the final visit. As a result, no eye was treated for OAG after vitrectomy throughout the follow-up period because the follow-up period might have been too short for late-onset OAG to develop. However, the upward trend and significant increase in IOP found in the MH group was notable.

The ever-increasing IOP changes in operated eyes in the $\mathrm{MH}$ group might to some extent be due to oxidation and alteration of the trabecular meshwork as previously reported because almost all eyes were underwent cataract surgery with vitrectomy [7]. However, we did not see IOP increases in the ERM group, although the postoperative lens status was similar between the ERM and MH groups in the current study.

The effects of residual TA should be taken into consideration. In the current study, TA was cleared intraoperatively as much as possible and IOP changes had shown trends to decrease rather than increase especially at 1 month after treatment. It was reported that intravitreal TA was cleared within 30 days to undetectable levels in vitrectomized rabbit eyes. Hence, the usage of TA as an adjunctive agent might not affect the IOP levels in long-term [14].

Therefore, we hypothesized that a difference in the postoperative inflammatory stress or the physical effect between the ERM and MH surgeries increased the concentrations of inflammatory cytokines or caused clogging of the trabecular meshwork with intraoperative sludge after fluid-gas exchange. The face-down position also could have been a potential cause.

Multivariate analysis revealed that significant risk factors were not so much gas tamponade, vitrectomy system, or 
adjunctive procedures but age and baseline IOP alone. On the other hand, Kaplan-Meier survival analysis with log-rank test between ERM and MH showed that the eyes in MH group had a higher risk of IOP increase than those in ERM group, and thus gas tamponade could have a possibility of IOP increase in long-tem. Recently, Ki-I et al. reported about long-term IOP changes after combined cataract surgery and vitrectomy in ERM or MH [15]. In their report, gas tamponade was not affected postoperative IOP and they concluded that the IOP change after phacovitrectomy may be limited and some case showed IOP increase exceeding baseline or fellow eyes in long-term, but they did not performed multivariate analysis. Our results were consistent with the report by Ki-I et al. at a point. It is inadequate for a definitive conclusion from these results because one possible reason could be refractive errors or axial lengths as the risk factors of open-angle glaucoma. It is well known that high myopia is one of the risk factors of OAG [16-18]. Further investigation is warranted with adjusted comorbid risk factors of OAG.

Our observations should be confirmed by further studies, because the current sample sizes were small, the study was retrospective, and the IOP was not measured using Goldmann applanation tonometry but non-contact tonometer, which could be affected by the corneal thickness. However, the IOP levels measured with non-contact tonometer are adoptable when the IOP levels are normotensive [19].

In conclusion, the current data suggested the risk of increasing IOP and possible development of late-onset OAG after MH surgery with gas tamponade. A new study with standardized IOP measurements, adjusted refractive errors, years of follow-up, a larger number of participants, and uniform definitions of glaucoma of OAG and OHT are needed to confirm this issue. Physicians should be aware of the potential risk of increased IOP after vitrectomy and provide treatment before irreversible visual impairment develops.

Open Access This article is distributed under the terms of the Creative Commons Attribution License which permits any use, distribution, and reproduction in any medium, provided the original author(s) and the source are credited.

\section{References}

1. Thompson JT, Sjaarda RN, Glaser BM, Murphy RP (1996) Increased intraocular pressure after macular hole surgery. Am J Ophthalmol 121:615-622
2. Chen PP, Thompson JT (1997) Risk factors for elevated intraocular pressure after the use of intraocular gases in vitreoretinal surgery. Ophthalmic Surg Lasers 28:37-42

3. Chen CJ (1998) Glaucoma after macular hole surgery. Ophthalmology 105:94-99, discussion 99-100

4. Costarides AP, Alabata P, Bergstrom C (2004) Elevated intraocular pressure following vitreoretinal surgery. Ophthalmol Clin N Am 17: 507-512

5. Tranos P, Asaria R, Aylward W, Sullivan P, Franks W (2004) Long term outcome of secondary glaucoma following vitreoretinal surgery. Br J Ophthalmol 88:341-343

6. Tranos P, Bhar G, Little B (2004) Postoperative intraocular pressure spikes: the need to treat. Eye 18:673-679

7. Chang S (2006) LXII Edward Jackson lecture: open angle glaucoma after vitrectomy. Am J Ophthalmol 141:1033-1043

8. Luk FO, Kwok AK, Lai TY, Lam DS (2009) Presence of crystalline lens as a protective factor for the late development of open angle glaucoma after vitrectomy. Retina 29:218-224

9. Yu AL, Brummeisl W, Schaumberger M, Kampik A, Welge-Lussen U (2010) Vitrectomy does not increase the risk of open-angle glaucoma or ocular hypertension-a 5-year follow-up. Graefe's archive for clinical and experimental ophthalmology $=$ Albrecht von Graefes Archiv fur klinische und experimentelle. Ophthalmologie 248: $1407-1414$

10. Lalezary M, Kim SJ, Jiramongkolchai K, Recchia FM, Agarwal A, Sternberg P Jr (2011) Long-term trends in intraocular pressure after pars plana vitrectomy. Retina 31:679-685

11. Holekamp NM, Shui YB, Beebe D (2006) Lower intraocular oxygen tension in diabetic patients: possible contribution to decreased incidence of nuclear sclerotic cataract. Am J Ophthal 141:1027-1032

12. Holekamp NM, Bai F, Shui YB, Almony A, Beebe DC (2010) Ischemic diabetic retinopathy may protect against nuclear sclerotic cataract. Am J Ophthalmol 150:543-550

13. Drance SM (1960) The significance of the diurnal tension variations in normal and glaucomatous eyes. Arch Ophthalmol 64: 494-501

14. Chin HS, Park TS, Moon YS, Oh JH (2005) Difference in clearance of intravitreal triamcinolone acetonide between vitrectomized and nonvitrectomized eyes. Retina 25:556-560

15. Ki IY, Yamashita T, Uemura A, Sakamoto T (2013) Long-term intraocular pressure changes after combined phacoemulsification, intraocular lens implantation, and vitrectomy. Jpn J Ophthalmol 57: $57-62$

16. Mitchell P, Hourihan F, Sandbach J, Wang JJ (1999) The relationship between glaucoma and myopia: the Blue Mountains Eye Study. Ophthalmology 106:2010-2015

17. Suzuki Y, Iwase A, Araie M, Yamamoto T, Abe H, Shirato S, Kuwayama Y, Mishima HK, Shimizu H, Tomita G, Inoue Y, Kitazawa Y (2006) Risk factors for open-angle glaucoma in a Japanese population: the Tajimi Study. Ophthalmology 113:16131617

18. Xu L, Wang Y, Wang S, Wang Y, Jonas JB (2007) High myopia and glaucoma susceptibility the Beijing Eye Study. Ophthalmology 114: 216-220

19. Choi WJ, Kim JW, Tchah H, Jin YH, Kim YJ (1990) Non-contact tonometry: an ideal method for mass screening. Korean J Ophthal: KJO 4:30-33 